\title{
Editorial
}

Wolfgang G. Stock und Christian Schlögl

\section{Gewachsene Kooperation der Düsseldorfer und Grazer Informationswissenschaft}

https://doi.org/10.1515/iwp-2017-0059

Liebe Leserinnen, liebe Leser,

im Wissenschaftsbetrieb sind derzeit Kooperationen sowohl im eigenen Hause als auch international - nicht nur üblich, sondern auch notwendig. Dies liegt zum großen Teil an der immer weiter voranschreitenden Spezialisierung in der Forschung. Arbeiten mehrere Spezialisten mit unterschiedlichen Hintergründen an einem Thema zusammen, ergeben sich (natürlich nur im positiven Fall) Synergieeffekte, die zu erfolgreichen Artikeln führen.

Auch kleinere Disziplinen wie die Informationswissenschaft sind von der Spezialisierung und der Internationalisierung der Forschung üblicherweise stark betroffen. Mit dem vorliegenden Themenheft wollen Kolleg/innen der Düsseldorfer und Grazer Informationswissenschaft die seit vielen Jahren bestehende Kooperation in Form von zwei gemeinsamen Schwerpunktheften der IWP zum Ausdruck bringen. Die Zusammenarbeit geht maßgeblich auf „alte“ Kontakte der Herausgeber zurück, als der eine als Lehrbeauftragter und der andere als wissenschaftlicher Mitarbeiter an der Uni Graz tätig waren. Die guten Kontakte aus den 1990er Jahren haben sich schließlich auf Institutsebene fortgesetzt und bestehen heute noch.

Die Idee zu dieser gemeinsamen Aktion wurde in einer Buschenschänke in Deutschlandsberg in der Steiermark geboren. Nachdem die beiden Herausgeber (und auch ihre jeweiligen Kollegen) in den letzten Jahren vorwiegend in Englisch publiziert hatten, kam nach ein, zwei Glas Schilcher der Gedanke auf, auch endlich einmal die deutschsprachige Informationswissenschaft und -praxis anzusprechen. Nach weiteren Gläsern fiel dann jede Bescheidenheit fort und wir entschieden uns dafür, unsere eigenen Hauptforschungsgebiete gebührend darzustellen.

Lassen Sie uns Ihnen nun eine kurze Einführung in dieses erste von zwei Themenheften geben. Der erste Beitrag von Isabelle Dorsch, Wolf Rauch und den Herausgebern (Forschungsthemen der Düsseldorfer und Grazer Informationswissenschaft) bietet einen Überblick über Forschungsinhalte der beiden Institute. $\mathrm{Zu}$ diesem Zweck bedient er sich einer szientometrischen Analyse, in der die Titelthemen der von 2010 bis 2016 erschienenen Publikationen analysiert wurden.
Ebenfalls eine Düsseldorfer Grazer Koproduktion ist der Beitrag von Lisa Beutelspacher, Stefan Dreisiebner und Maria Henkel (Informationskompetenz - Forschung in Graz und Düsseldorf). In ihm wird ein Überblick über an beiden Instituten durchgeführte Informationskompetenz-Studien gegeben. Schwerpunktmäßig geht es dabei um die Vermittlung und Messung von Informationskompetenz. Konkret wird ein Projekt vorgestellt, in dessen Rahmen ein mehrsprachiger Informationskompetenz-MOOC entwickelt wird.

Der Artikel von Leon Ladewig und Isabella Peters zeigt, dass szientometrische Methoden auch gut geeignet sind, um interdisziplinäre „Forschungssituationen “ zu vermessen. Konkret handelt es sich um den Exzellenzcluster „The Future Ocean“. Isabella Peters ist zwar mittlerweile am Leibniz-Informationszentrum Wirtschaft (ZBW) beschäftigt und unterrichtet als Professorin an der Uni Kiel, sie war aber zuvor viele Jahre an der Düsseldorfer Informationswissenschaft tätig.

Die beiden abschließenden Beiträge beschäftigen sich mit sehr aktuellen und gesellschaftlich brisanten Themen. Im ersten untersuchen Katrin Scheibe, Franziska Zimmer und Kaja J. Fietkiewicz das Informationsverhalten von „Streamern“ und Zuschauern beim Social Live Streaming Dienst YouNow. Unter anderem werden die Motive der Streamer, die angebotenen Inhalte sowie begangene Rechtsverletzungen aufgezeigt.

Der zweite Beitrag (Informationswissenschaft in der Urbanistik) wurde von einem Düsseldorfer Autorenkollektiv verfasst. Ihm liegt ein umfassendes Forschungsprojekt zugrunde, dessen Ziel darin besteht zu erforschen, wie die informationelle/smarte Stadt als prototypische Stadt der aufkommenden Wissensgesellschaft aussieht bzw. konkret gestaltet sein sollte. Im ersten Teil dieses Aufsatzes werden der konzeptionelle Forschungsrahmen und die verwendeten Forschungsmethoden vorgestellt. Der zweite Teil mit den ersten empirischen Ergebnissen erscheint im nächsten Heft der IWP.

Viel Spaß beim Lesen des einen oder anderen Artikels wünschen Ihnen

Wolfgang G. Stock und Christian Schlögl 